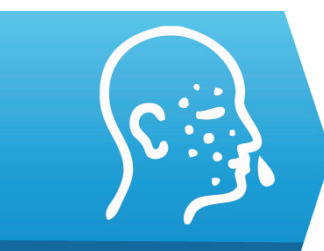

IMMUNOLOGY

\title{
Successful desensitization in a patient with hypersensitivity to multiple insulin preparations - case report
}

\author{
Carina Petrică $u^{1,2}$, Irena Nedelea ${ }^{1,2}$, Diana Deleanu ${ }^{1,2,3}$
}

1) Allergy Outpatient Unit, Professor Doctor Octavian Fodor Regional Institute of Gastroenterology and Hepatology Cluj-Napoca, Romania

2) Department of Allergology and Immunology, Iuliu Hatieganu University of Medicine and Pharmacy, Cluj-Napoca, Romania

3) Internal Medicine Department, Professor Doctor Octavian Fodor Regional Institute of Gastroenterology and Hepatology, Cluj-Napoca, Romania

DOI: $10.15386 / \mathrm{mpr}-1643$

Manuscript received: 04.04.2020

Received in revised form: 06.08 .2020

Accepted: 27.08.2020

Address for correspondence:

irennedelea@gmail.com

This work is licensed under a Creative Commons Attribution-NonCommercialNoDerivatives 4.0 International License

\begin{abstract}
Hypersensitivity to insulin has decreased substantially in the past three decades, since purified human insulin was introduced to replace heterogeneous porcine and bovine preparations. However, human insulin and its analogs still have immunogenic potential that may prove detrimental for hypersensitive insulin-dependent diabetics. In cases of anaphylactic reactions to insulin, rapid desensitization may be considered as a treatment strategy. We present the first case of successful insulin desensitization in Romania for an uncontrolled diabetic patient with type I hypersensitivity to multiple insulin analogs.
\end{abstract}

Keywords: insulin, drug allergy, hypersensitivity, immunotherapy

\section{Introduction}

Hypersensitivity to insulin has decreased substantially in the past three decades since purified human insulin was introduced to replace heterogeneous porcine and bovine preparations [1]. However, human insulin and its analogs still pose immunogenic potential which can prove detrimental for hypersensitive patients with diabetes requiring insulin treatment [2]. According to the latest relevant epidemiological studies [3] insulin allergy incidence varies from 0.1 to $3 \%$. Less than one third of reactions are considered to be linked to insulin itself, while the remainder occur due to additives (protamine, zinc, and cresol) found in the preparations [4]. These reactions are most commonly represented by immediate IgE-mediated (type I) reactions, clinically ranging from local injection site erythema to severe generalized anaphylaxis, but type III (immune-complex mediated) and type IV (delayed cell mediated) reactions can also be involved, clinically represented by subcutaneous nodules and late-phase cutaneous reactions respectively $[1,3]$. Considering that local reactions can precede systemic anaphylactic episodes [5], desensitization is a necessary safety precaution therapeutic measure for patients with diabetes requiring insulin treatment.

To our knowledge, we present the first case of successful insulin desensitization in Romania along with the recommended protocol for a patient with uncontrolled diabetes presenting a type I hypersensitivity to multiple insulin analogs.

\section{Case report}

A 49-year-old overweight, hypertensive female patient, from an urban environment, diagnosed with type 2 diabetes complicated with diabetic sensory peripheral neuropathy and nonproliferative diabetic retinopathy was initially treated with triple therapy of oral hypoglycemic agents which were unable to control her glycemia, thus insulin was introduced. She presented with a history of repeated extensive local reactions to various insulin preparations. Insulin aspart was discontinued after three weeks of repeated local erythema and edema 90 minutes after subcutaneous administration. The following year, an attempt to introduce insulin glargine was made, however the patient developed an extensive local reaction again within the first 90 minutes of administration. 
In the mean time, the patient was treated with oral hypoglycemic agents and subcutaneous GLP1 analog exenatide (initially one preparation administered $5 \mathrm{mcg}$ twice daily then another preparation $2 \mathrm{mg}$ once a week), which surprisingly also led to delayed extensive local reactions after each injection, but were continued for a year along with antihistamine premedication. Because the diabetes was uncontrolled, an attempt to introduce insulin lispro and insulin glulisine was made only to observe the recurrence of large local reactions. The patient was referred to the Allergy clinic for further investigations and possible therapeutic options. Written informed consent for all procedures and photos was obtained from the patient.

Immunological evaluations revealed the following: (1) Skin prick tests were negative to insulin glulisine, latex and atopic panel; (2) Intradermal test with a diluted concentration of $1 / 100(1 \mathrm{U} / \mathrm{ml})$ insulin glulisine was positive within an hour; (3) Patch tests to insulin glulisine, latex and zinc were negative at 48 and 96 hours; (4) Insulin specific-IgE was negative; (5) Due to financial constraints, basophil degranulation test to insulin was not possible.

Based on positive personal history of repeated extensive local reactions and demonstrated cutaneous sensitivity to insulin, desensitization to insulin glulisine was initiated. We started the rapid desensitization protocol with progressive dose-escalation of subcutaneously administered insulin glulisine with a target dose of 8 units (Table I). Because no international consensus upon a desensitization protocol for insulin has been published to date, we established the protocol used in this case based on clinical experience. The start dose was $0.000001 \mathrm{U}$, and doses were escalated every 30 minutes. Within 10 minutes of administering the $0.01 \mathrm{U}$ dose (the $5^{\text {th }}$ administration), the patient presented a local reaction with pruritus, erythema and $12 \mathrm{~mm}$ diameter edema. In case of immediate local reactions, the same dose was repeated at 30 min intervals until no reaction occurred. We continued increasing the dose twofold, until we reached a dose of 4 UI (cumulative dose of $7.231111 \mathrm{U}$ ). All the injection sites presented with a local wheal and flare reaction approximately 1 hour after the last administration, and 6 hours after the beginning of the desensitization (Figure 1A). Moreover, the patient presented extensive induration and erythema of injection sites approximately 12 hours after injection which lasted for 24 hours (Figure 1B). The following day, premedication with $10 \mathrm{mg}$ Desloratidine one hour before the injection and $10 \mathrm{mg}$ Montelukast in the evening was initiated. In addition, we changed the site of administration. 8 UI maintenance daily dose was reached on the fourth day, without any further adverse reactions.

At the 1 month check-up appointment, the patient was tolerating the insulin glulisine injections. However, she presented palmar eczema of unknown origin. The dermatologist recommended emollient and reepithelization creams which quickly resolved the eczema. Because insulin glulisine (rapid meal time insulin) was not controlling blood glucose enough, a long acting insulin (diabetologist suggested insulin glargine) was needed. Considering the well tolerated initial insulin preparation we used a desensitization protocol starting with a higher initial dose, being able to introduce 12 units of insulin glargine in the patient's regimen as well (Table II). Skin testing to insulin glargine prior to initiating the desensitization was negative.

Table I. Desensitization protocol to Insulin glulisine.

\begin{tabular}{|c|c|c|c|c|c|c|c|}
\hline Day & $\begin{array}{c}\text { Dose } \\
\text { number }\end{array}$ & Units/dose & $\begin{array}{c}\text { Cumulative dose } \\
\text { (units) }\end{array}$ & Site & $\begin{array}{l}\text { Immediate local } \\
\text { reaction }\end{array}$ & $\begin{array}{l}\text { Late local } \\
\text { reaction }\end{array}$ & $\begin{array}{l}\text { Systemic } \\
\text { reaction }\end{array}$ \\
\hline 1 & 1 & 0.000001 & 0.000001 & Abdomen & No & Yes & No \\
\hline 1 & 2 & 0.00001 & 0.000011 & Abdomen & No & Yes & No \\
\hline 1 & 3 & 0.0001 & 0.000111 & Abdomen & No & Yes & No \\
\hline 1 & 4 & 0.001 & 0.001111 & Abdomen & No & Yes & No \\
\hline 1 & 5 & 0.01 & 0.011111 & Abdomen & Yes & Yes & No \\
\hline 1 & 6 & 0.01 & 0.021111 & Abdomen & Yes & Yes & No \\
\hline 1 & 7 & 0.01 & 0.031111 & Abdomen & No & Yes & No \\
\hline 1 & 8 & 0.1 & 0.131111 & Abdomen & Yes & Yes & No \\
\hline 1 & 9 & 0.1 & 0.231111 & Abdomen & No & Yes & \\
\hline 1 & 10 & 1 & 1.231111 & Abdomen & No & Yes & No \\
\hline 1 & 11 & 2 & 3.231111 & Abdomen & No & Yes & No \\
\hline 1 & 12 & 4 & 7.231111 & Abdomen & No & Yes & No \\
\hline 2 & 13 & 2 & 2 & Leg & No & No & No \\
\hline 2 & 14 & 4 & 6 & Leg & No & No & No \\
\hline 3 & 15 & 6 & 6 & Leg & No & No & No \\
\hline 4 & 16 & 8 & 8 & Leg & No & No & No \\
\hline
\end{tabular}




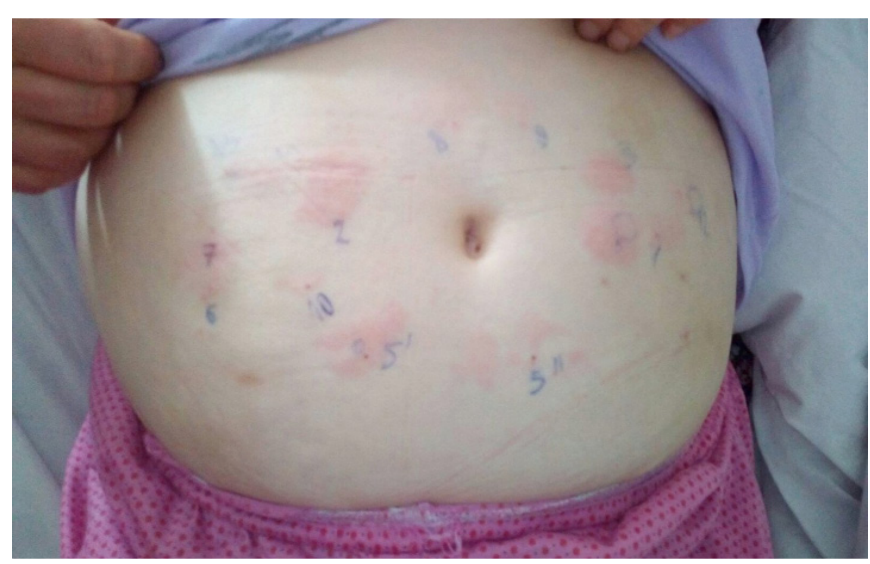

A

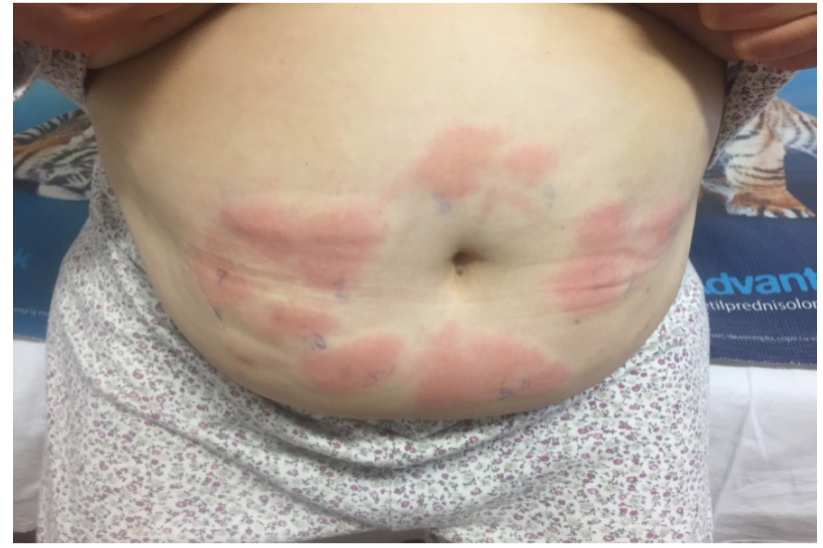

B

Figure 1. A. Immediate wheal and flare reaction 1 hour after insulin glulisine injections. B. Late phase reaction with induration and erythema 24 hours after insulin glulisine injections.

Table II. Desensitization protocol to insulin glargine.

\begin{tabular}{l|c|c|c|c|cc} 
Day & Dose number & Units/dose & $\begin{array}{c}\text { Cumulative dose } \\
\text { (units) }\end{array}$ & $\begin{array}{c}\text { Immediate local } \\
\text { reaction }\end{array}$ & $\begin{array}{c}\text { Late local } \\
\text { reaction }\end{array}$ & Systemic reaction \\
\hline $\mathbf{1}$ & 1 & 2 & 2 & No & No & No \\
$\mathbf{1}$ & 2 & 4 & 6 & No & No & No \\
$\mathbf{2}$ & 3 & 4 & 4 & No & No & No \\
$\mathbf{2}$ & 4 & 6 & 10 & No & No & No \\
$\mathbf{3}$ & 5 & 10 & 10 & No & No & No \\
$\mathbf{4}$ & 6 & 12 & 12 & No & No & No
\end{tabular}

Based on the clinical context, the positive intradermal test to insulin and favorable response to desensitization, we suspect a type-I hypersensitivity reaction in the presented case. Aside from type I hypersensitivity reactions, two other pathological mechanisms can explain recurrent local reactions to insulin [6]. Type III (Arthus) hypersensitivity reactions due to insulin: IgG anti-insulin immune complexes which appear 4 to 6 hours after injection while type IV (delayed-cell mediated) hypersensitivity reactions peak at 24 to 48 hours after injection. As mentioned above, similar local reactions were observed in our patient with the injection of GLP-1 analog exenatide, which raises the question if this patient is also sensitized to exenatide or to additives found in the preparations [7]. Cresol is found in all the insulin preparations tested on this patient as well as in the GLP-1 analog exenatide preparations. Since cresol extract was not available for cutaneous testing, we could not investigate for a possible sensitization.

Management of insulin allergy includes attempting oral hypoglycemic agents, injections with glucagon-like peptide 1 analogs, antihistamines, and switching to a different insulin preparation [1,3]. However, when the above measures are not feasible due to multiple sensitizations or insufficient control of blood glucose, desensitization is the most suitable option [8]. Even though the mechanisms of inducing immunological tolerance through desensitization are not fully elucidated, in order to maintain the state of anergy and not loose tolerance the drug must be administered continuously [9], in our case, daily.

\section{Acknowledgements}

We thank Dr. Adela Teodorovici, Dr. Laura Fekete, and Dr. Maria Ardel Mihalca for their help during the desensitization procedure.

\section{References}

1. Heinzerling L, Raile K, Rochlitz H, Zuberbier T, Worm M. Insulin allergy: clinical manifestations and management strategies. Allergy. 2008;63:148-155.

2. Fernández L, Duque S, Montalbán C, Bartolomé B. Allergy to human insulin. Allergy. 2003;58:1317.

3. Haastrup MB, Henriksen JE, Mortz CG, Bindslev-Jensen C. Insulin allergy can be successfully managed by a systematic 
approach. Clin Transl Allergy. 2018;8:35.

4. Ghazavi MK, Johnston GA. Insulin allergy. Clin Dermatol. 2011;29:300-305.

5. Eguíluz-Gracia, Rodríguez-Alvarez M, Cimarra-Alvarez M, Sanabria-Pírez MC, Martínez-Cócera C. Desensitization for insulin allergy: a useful treatment also for local forms. J Investig Allergol Clin Immunol. 2012;22:215-216.

6. deShazo RD, Boehm TM, Kumar D, Galloway JA, Dvorak HF. Dermal hypersensitivity reactions to insulin: correlations of three patterns to their histopathology. J Allergy Clin Immunol. 1982;69:229-237.
7. Steveling EH, Winzeler B, Bircher AJ. Systemic Allergic Reaction to the GLP-1 Receptor Agonist Exenatide. J Pharm Technol. 2014;30:182-186.

8. Wu P, Ji C, Wang M, Zou S, Ge W. Desensitization of allergy to human insulin and its analogs by administering insulin aspart and insulin glargine. Ann Endocrinol (Paris). 2013;74:56-58.

9. Cernadas JR, Brockow K, Romano A, Aberer W, Torres MJ, Bircher A, et al. General considerations on rapid desensitization for drug hypersensitivity - a consensus statement. Allergy. 2010;65:1357-1366. 